\title{
Commentary: Leveling up the evidence!
}

\author{
Konrad Hoetzenecker, MD, PhD
}

\author{
From the Division of Thoracic Surgery, Medical University of Vienna, Vienna, Austria. \\ Disclosures: Author has nothing to disclose with regard to commercial support. \\ Received for publication Aug 1, 2019; accepted for publication Aug 1, 2019; available ahead of print Oct 13, \\ 2019. \\ Address for reprints: Konrad Hoetzenecker, MD, PhD, Division of Thoracic Surgery, Medical University of \\ Vienna, Waehringer Guertel 18-20, 1090 Vienna, Austria (E-mail: konrad.hoetzenecker@meduniwien.ac.at). \\ J Thorac Cardiovasc Surg 2020;159:731 \\ $0022-5223 / \$ 36.00$ \\ Copyright (C 2019 by The American Association for Thoracic Surgery \\ https://doi.org/10.1016/j.jtcvs.2019.08.011
}

I read with great interest the article "Outcomes of Marginal Donors for Lung Transplantation After Ex Vivo Lung Perfusion: A Systematic Review And Meta-analysis," published by Nakajima and colleagues ${ }^{1}$ in this issue of the Journal. It provides a summary of published evidence comparing outcomes of marginal donor lungs pretreated by ex vivo lung perfusion (EVLP) to outcomes of standard donor lungs preserved by static cold storage. A total of 8 studies with nearly 1200 patients met the inclusion criteria and served as the basis for a meta-analysis. The central message of this work is that EVLP-treated marginal lungs perform equally well as standard donor lungs.

To the best of my knowledge this is the first meta-analysis of ex vivo lung perfusion. The fact that this analysis was published in the Journal of Thoracic and Cardiovascular Surgery highlights the high reputation that the Journal currently enjoys in the lung transplant community.

Performing EVLP for marginal lungs has 2 major advantages: First, it serves as an additional safety net, allowing lungs to be observed under "near physiologic" conditions for 4 to 6 hours. $^{2}$ Second, there is emerging evidence that the perfusion itself can improve damaged grafts and render them into a transplantable state. ${ }^{3,4}$ Especially the first indication - the use of EVLP as a safety net-has lately gained increasing importance. Most transplant centers nowadays accept marginal donor lungs on a routine basis. In the clinical reality, however, it is often unclear how marginal donor lungs can be in order to still to be used safely for transplantation. We have recently shown that organs that had previously been rejected by 2 or more transplant centers for quality reasons can often be used without hampering long-term outcome. ${ }^{5}$ The use of EVLP to determine the true functional quality of such grafts adds an important tool in the armamentarium of lung transplant surgeons. Another example is the use of donation-after-circulatory-death lungs from donors with a prolonged time between withdrawal of life-sustaining treatment and arrest. Potential graft damage resulting from prolonged warm ischemic times can be examined

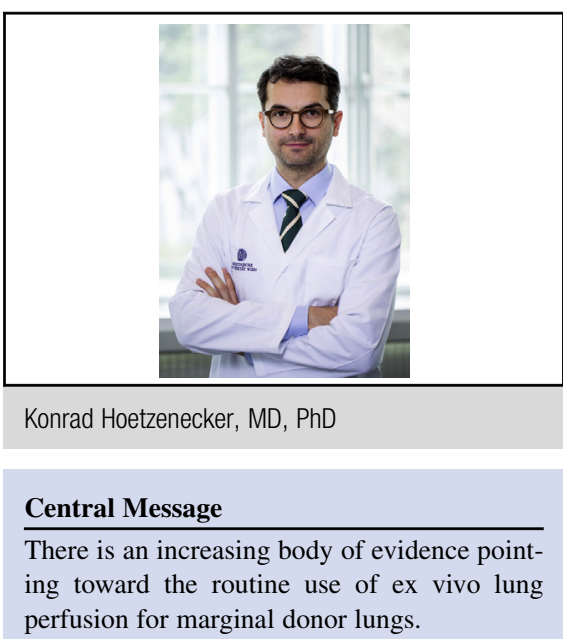

See Article page 720.

during EVLP, and often those lungs turn out to be acceptable for transplantation. ${ }^{6}$

The growing evidence of EVLP emphasizes the central place of this technique in contemporary lung transplantation. This meta-analysis performed by the Tokyo group $^{1}$ is highly relevant. It provides high-level evidence favoring the use of EVLP for marginal donor lungs. Compared with static cold storage, EVLP platforms are extremely costly, but they facilitate the use of organs otherwise lost for the organ pool. Analyses such as that of Nakajima and colleagues ${ }^{1}$ provide indispensable arguments when discussing the costs of an EVLP program with hospital authorities and management boards.

\section{References}

1. Tian D, Wang Y, Shiiya H, Sun C-B, Uemura Y, Sato M, et al. Outcomes of marginal donors for lung transplantation after ex vivo lung perfusion: a systematic review and meta-analysis. J Thorac Cardiovasc Surg. 2020;159:720-30.e6.

2. Cypel M, Yeung JC, Machuca T, Chen M, Singer LG, Yasufuku K, et al Experience with the first 50 ex vivo lung perfusions in clinical transplantation. J Thorac Cardiovasc Surg. 2012;144:1200-6.

3. Aigner C, Slama A, Hötzenecker K, Scheed A, Urbanek B, Schmid W, et al Clinical ex vivo lung perfusion-pushing the limits. Am J Transplant. 2012;12: 1839-47.

4. Sage E, Mussot S, Trebbia G, Puyo P, Stern M, Dartevelle P, et al. Lung transplantation from initially rejected donors after ex vivo lung reconditioning: the French experience. Eur J Cardiothorac Surg. 2014;46:794-9.

5. Schwarz S, Muckenhuber M, Benazzo A, Glueck O, Evermann M, Beer L, et al Outcome after lung transplantation using multiple declined marginal donor lungs. Transplant Int. 2018:31(Suppl S4):13.

6. Reeb J, Keshavjee S, Cypel M. Successful lung transplantation from a donation after cardiocirculatory death donor taking more than 120 minutes to cardiac arrest after withdrawal of life support therapies. J Heart Lung Transplant. 2016;35: 258-9. 